\title{
Nucleotide variation in Quercus crispula Blume
}

\author{
ND Quang, S Ikeda and K Harada \\ Faculty of Agriculture, Ehime University, Tarumi, Matsuyama, Japan
}

\begin{abstract}
Quercus is attractive for evolutionary studies, primarily for developing the concepts of the species, speciation and adaptation; however, remarkably little is known about levels of nucleotide polymorphism in the nuclear functional genes of this genus. This article provides the first characterization of levels of nucleotide polymorphism in 11 gene fragments in natural populations of a Quercus species, Quercus crispula Blume. Results show that the level of nucleotide variation in this oak is generally higher than that in conifers, as high as that in a European oak, but lower than that in an aspen. The level of population recombination is relatively high. Withinpopulation inbreeding is negligible and between-population
\end{abstract}

differentiation is modest. The decay of linkage disequilibrium is significantly faster in the species-wide samples and the three northernmost populations than in the other populations. Statistical tests support the hypothesis of a recent bottleneck for several populations in the southern part of Japan. The amounts and patterns of nucleotide variation, recombination and linkage disequilibrium, and genetic differentiation observed among populations of this species are contradictory to our expectations, given the recent colonization history of the northern Japan populations.

Heredity (2008) 101, 166-174; doi:10.1038/hdy.2008.42; published online 28 May 2008

Keywords: oak; nucleotide variation; DNA; evolution; Quercus; genetics

\section{Introduction}

Nucleotide sequence data integrate information over a long time period (on average, $4 N$ generations, where $N$ is the effective population size), and thus can provide powerful insights into the important factors contributing to the evolutionary dynamics of a species. In any natural population, the amounts and patterns of nucleotide variation are influenced by both biological factors (for example, mutation, recombination and natural selection) and demographic factors (for example, range expansion and colonization, population subdivision and admixture) affecting $N$.

In recent years, nucleotide variation in nuclear functional genes has been extensively studied in herbaceous plants (Innan et al., 1996; Filatov and Charlesworth, 1999; Kuittinen and Aguage, 2000; Aguade, 2001; Zhang and Ge, 2006; Arunyawat et al., 2007). However, there has been only a very limited number of such published reports about tree species. The amounts and patterns of genetic variation in plants are strongly affected by life history traits and mating systems (Hamrick and Godt, 1996); hence, it will be problematic to generalize the patterns of nucleotide variation in herbaceous plants to woody plants. Thus far, most data of nucleotide variation in trees have been from Pinus (Dvornyk et al., 2002; Garcia-Gil et al., 2003; Neale and Savolainen, 2004; Pot et al., 2005), Cryptomeria (Kado et al., 2003), Chamaecyparis (Kado et al., 2008) and Populus (Ingvarsson, 2005).

Correspondence: Dr ND Quang, Faculty of Agriculture, Ehime University, 3-5-7 Tarumi, Matsuyama, Japan.

E-mail: quanghoa@agr.ehime-u.ac.jp.

Received 22 January 2008; revised 10 April 2008; accepted 15 April 2008; published online 28 May 2008
The genus Quercus comprises 350-500 species distributed throughout the northern hemisphere (Kubitzki, 1993; Nixon, 1993). They are conspicuous members of temperate deciduous forests of North America, Europe, Asia, as well as the evergreen Mediterranean maquis. The members of this genus are attractive for research, primarily for developing concepts of the species, speciation and evolution (Van, 1976; Rieseberg and Wendel, 1993). Nonetheless, remarkably little is known about levels of nucleotide polymorphism in the functional genes of this genus. Hence, this article provides the first characterization of levels of nucleotide polymorphism in a species of Quercus, Quercus crispula Blume.

$Q$. crispula Blume (Fagaceae) is a common tree throughout the cool-temperate deciduous forests of southern Sakhalin, the Kuril Islands, Japan, and Korea (Watanabe, 1994). The populations are dense and continuous in northern Japan but sparse and commonly isolated in small patchy forests at elevations $>800 \mathrm{~m}$ in the south (Figure 1). cpDNA data (Kanno et al., 2004; Okaura et al., 2007) are consistent with palynological data (Hibino and Takeuchi, 1998; Miyoshi, 1998; Morita et al., 1998), suggesting a recent colonization history (from 8000 to 12000 years ago) for the northern Japan (approximately more than $35^{\circ} \mathrm{N}$ ) populations. The southern populations are shown to have remained in situ during the last glacial maximum (LGM) and lasted from 20000 to 18000 years BP. The former populations are likely to be adaptive to a colder climate and the latter to a warmer climate (Okaura et al., 2007). Our previous cpDNA study also revealed a similar colonization history for another deciduous tree, Fagus crenata (Fagaceae), usually forming a major vegetation class together with $Q$. crispula (Okaura and Harada, 2002).

The present study addresses the following questions: (1) What is the level of nucleotide diversity in this oak 
species as compared to pines and other broadleaved species? (2) What are the pattern and extent of linkage disequilibrium (LD) among populations of $Q$. crispula? (3) Do the patterns of genetic variation differ significantly

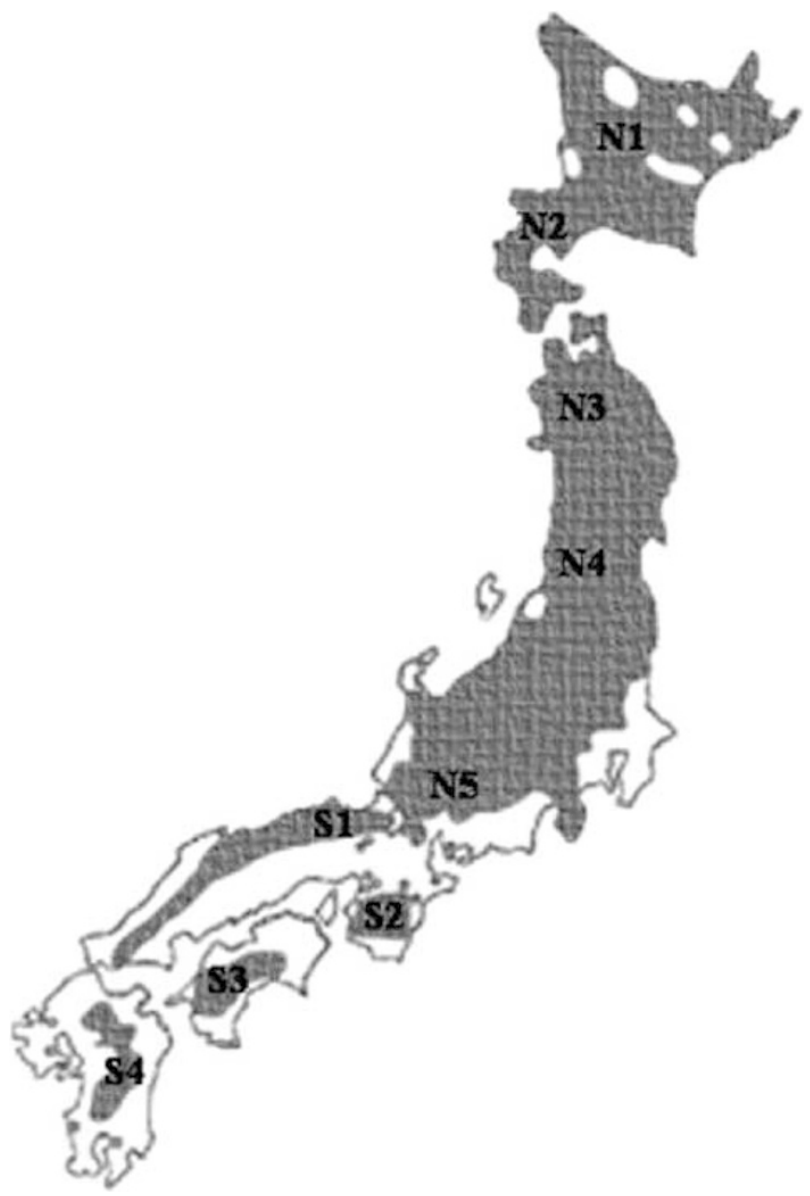

Figure 1 Approximate geographical distribution of the populations sampled and the current pattern of species distribution in Japan. The name codes of each population (N1-S4) are the same as those given in Supplementary Table S1. The shadings indicate the patterns of the current population distribution and density, based on Horikawa (1972). between the northern and southern Japan populations? (4) Can we find sequence evidence favoring the hypothesis of climatic adaptation in populations of $Q$. crispula?

\section{Materials and methods}

\section{Samples}

Fresh leaves were collected from trees belonging to nine populations of $Q$. crispula distributed throughout Japan (Figure 1; Supplementary Table S1). Leaves collected from another Japanese oak were used as outgroup species (Quercus phillyraeoides, no morphological evidence for the hybridization of this species with $Q$. crispula). We collected leaves from trees growing at least $30 \mathrm{~m}$ apart. Trees clearly distinguishable by morphological characteristics were chosen. The samples were immediately processed for DNA extraction or frozen at $-20{ }^{\circ} \mathrm{C}$ until use.

\section{Loci}

We genotyped 11 loci, all of which are nuclear functional genes (Table 1). Except for MetE, the others are 10 expressed sequence tag (EST) loci extracted from oak EST resources reported in a previous study (Casasoli et al., 2006).

\section{DNA extraction and PCR amplification}

Total genomic DNA was extracted from fresh or frozen leaf tissues using the cetyltrimethylammonium bromide method. The primers for the amplification of MetE are identical to those specified by Chiang et al. (2002). Those for the amplification of the 10 EST loci are the same as those reported by Casasoli et al. (2006).

\section{Subcloning and sequencing}

The three long (>1000 bp) loci, MetE, Cons109 and Cons14, were cloned and sequenced to generate haplotype sequences. The PCR products were cloned into the TOPO vector using a Zero Blunt TOPO PCR Cloning kit for sequencing (Invitrogen). The clones were sequenced using the BigDye terminator sequencing kit (Applied Biosystems, Foster City, CA, USA) on an ABI310 automated sequencer (Applied Biosystems). At least

Table 1 Summary of loci studied

\begin{tabular}{|c|c|c|c|c|c|}
\hline \multirow[t]{2}{*}{$\overline{\text { Locus }}$} & \multirow[t]{2}{*}{ Location $^{\text {a }}$} & \multicolumn{3}{|c|}{ Alignment length $(b p)$} & \multirow[t]{2}{*}{ Functional association $^{\mathrm{b}}$} \\
\hline & & Total & Exon & Intron & \\
\hline MetE & NA & 1385 & 611 & 774 & Catalyst in the terminal step in the methionine biosynthesis of plants \\
\hline Cons21 & Q2 & 480 & 179 & 301 & Arabidopsis thaliana ribosomal protein S27 $\left(4 \mathrm{e}^{-40}\right)$ \\
\hline $01 \mathrm{~A} 03$ & Q77 & 539 & 212 & 327 & Fragaria $\times$ ananassa putative $40 S$ ribosomal protein $\left(\mathrm{e}^{-126}\right)$ \\
\hline Cons126 & $\hat{\mathrm{Q}} 7$ & 239 & 188 & 51 & Prunus avium 60 S ribosomal protein L24 $\left(3 \mathrm{e}^{-62}\right)$ \\
\hline Cons107 & Q11 & 448 & 222 & 226 & Alnus firma mRNA for nonsymbiotic hemoglobin $\left(2 \mathrm{e}^{-76}\right)$ \\
\hline Cons58 & Q5 & 240 & 110 & 130 & Arabidopsis thaliana dormancy-associated protein $\left(4 \mathrm{e}^{-22}\right)$ \\
\hline Cons105 & Q12 & 440 & 90 & 350 & Malus domestica transposon gene for putative DNA topoisomerase $\left(5 \mathrm{e}^{-45}\right)$ \\
\hline Cons41 & NA & 354 & 244 & 110 & Aquilegia formosa heat shock protein $70\left(\mathrm{HSP} 70 ; 3 \mathrm{e}^{-60}\right.$ ) \\
\hline Cons106 & NA & 237 & 189 & 48 & Spinach gene for plastocyanin $\left(7 \mathrm{e}^{-27}\right)$ \\
\hline Cons14 & Q5 & 1100 & 243 & 857 & Arabidopsis thaliana At2g34480 gene $\left(4 \mathrm{e}^{-36}\right)$ \\
\hline Cons109 & Q7 & 1015 & 194 & 821 & Fagus sylvatica mRNA for metallothionein-like protein $\left(\mathrm{e}^{-100}\right)$ \\
\hline Total & $\geqslant 5$ & 6477 & 2045 & 4432 & \\
\hline
\end{tabular}

${ }^{a}$ Chromosome location of the 10 EST loci are the same as in Casasoli et al. (2006); NA, not yet mapped.

${ }^{\mathrm{b}}$ The sequence of 10 EST loci of Quercus crispula was used to search for homologous gene by BLAST tool in DDBJ (http:// www.ddbj.nig.ac.jp/); numbers in the parentheses are $E$-values. 
8-10 different clones of each fragment were sequenced to detect the presence of multiple haplotypes within individuals and to control for Taq polymerase errors. For the other eight short $(<600 \mathrm{bp}$ ) loci, we used a direct sequencing technique to obtain single nucleotide polymorphism (SNP) data. The frequency of each SNP was scored by direct sequencing of the gene fragments in which it was present. Heterozygous sites in a single individual were visible as double peaks in the chromatograms generated by the automated sequencer. The peaks were confirmed by repeating the experiment twice if necessary.

\section{Data analysis}

Sequences were verified manually. All indels (insertion/ deletion) were excluded from analyses. Analyses of sequence data of the three long loci, MetE, Cons109 and Cons14, were performed using DnaSP version 4.10.8 (Rozas et al., 2003). For the eight short loci, nucleotide diversity $(\pi)$ was estimated by dividing the average pairwise sequence difference $(k)$ by the number of sites.

The alignments of all the sequences of MetE, Cons109 and Cons 14 were used to perform analysis of recombination with DnaSP. The minimum number of recombination events $\left(R_{\mathrm{M}}\right)$ that have occurred along the sequence was estimated following the four-gamate test (Hudson and Kaplan, 1985). The two indices of LD, $D^{\prime}$ and $r^{2}$, were estimated between pairs of polymorphic sites for each locus with DnaSP. The statistical significance of each pairwise test of LD was evaluated using the $\chi^{2}$-test. All pairwise analyses are not fully independent because of LD itself; thus, the proportion of significant $(P<0.05)$ pairwise tests was compared for pairs of sites separated by different physical distances ( $>400$ or $<400 \mathrm{bp}$ ). Comparison of these ratios over the two separate distance ranges was carried out using Fisher's exact test (two-tailed). Although the nonlinear regression analysis (Remington et al., 2001) was useful as a representation of the overall LD rate (Ingvarsson, 2005; Zhu et al., 2007), it yields a least-squares estimate of the population recombination rate per base pair; this estimate may be imprecise and unrealistic because of several factors, for example, the nonindependence between linked sites and the nonequilibrium populations.

Tajima's $D$ (Tajima, 1989a) statistic was used to test for deviations from neutrality of the three loci, MetE, Cons109 and Cons14. This test measures skews in the frequency spectrum; a negative $D$ suggests an excess of low-frequency polymorphisms and a positive $D$ indicates an excess of intermediate-frequency polymorphisms. Tajima's test is conservative for testing departures from neutral equilibrium conditions, particularly under the assumption of no recombination. In addition, the test statistics $D$ and F proposed by $\mathrm{Fu}$ and $\mathrm{Li}$ (1993) were computed for the three loci. All these tests assume random mating and random sampling, and DnaSP was used to carry out the tests. We did not apply these tests for the eight short loci because of the small number of segregating sites per locus. Instead, we performed the multilocus Hudson-Kreitman-Aguade (HKA) test (Hudson et al., 1987; http://lifesci.rutgers.edu/ heylab/) to assess the neutral prediction of the ratio of polymorphism to divergence across the eight EST loci. $Q$. phillyraeoides homologous sequences were used as outgroup for this test.
Inbreeding indices $\left(\mathrm{F}_{\mathrm{IS}}\right)$ and their statistical significance (with 1000 randomizations) were estimated using FSTAT ver. 2.9.3.2 (Goudet, 2001). The SNP data of all the 11 loci were used in this analysis for the three northernmost and three southernmost populations; SNP data of the three loci, MetE, Cons109 and Cons14, were used for estimating $\mathrm{F}_{\mathrm{IS}}$ for the three central populations (N4-S1). We estimated the standard $\mathrm{F}_{\mathrm{ST}}$ statistic for MetE, Cons109 and Cons14 using the method of Hudson et al. (1992); the probability of the statistical test of genetic differentiation was estimated using the method of Hudson (2000) (Snn statistic-based probabilities, evaluated by 10000 permutations). For the eight short loci, we used their SNP data to estimate $\mathrm{F}_{\mathrm{ST}}, 99 \%$ confidence intervals of $\mathrm{F}_{\mathrm{ST}}$, and its statistical significance with 1000 randomizations with FSTAT.

\section{Results}

We obtained the sequences of 11 loci from three northernmost (N1-N3) and three southernmost (S2-S4) populations; the sequences of MetE, Cons109 and Cons14 were additionally obtained from three populations (N4, N5 and S1) located approximately in the center of Japan (Figure 1). The length of the aligned sequence for each locus varied between 237 and $1385 \mathrm{bp}$, with a total of $6477 \mathrm{bp}$ in length. The number of sequences sampled for each locus $(n)$ in each population is shown in Tables 2 and 3. Only two variants were detected for all the polymorphic sites in this study. A total of 261 SNPs were detected, and thus an average of 1 SNP every $\sim 25 \mathrm{bp}$ was found in our sample set. High haplotype fractions $(\mathrm{h} / \mathrm{n})$ were found in every population at all loci (Table 2$)$.

\section{Levels of within-population inbreeding and \\ between-population differentiation}

The $F_{I S}$ values did not vary significantly among different populations, ranging from -0.081 (N5) to 0.044 (S1). None of the populations examined had $F_{I S}$ values significantly deviating from 0 . The $F_{S T}$ values are summarized in Table 3 . There was slight variation across loci in the levels of genetic differentiation. Although all loci showed significant genetic differentiation across populations, the level of population differentiation was modest. The averages of $\mathrm{F}_{\mathrm{ST}}$ across loci were low both in the pool and in the three northernmost and three southernmost populations (Table 3). Previous microsatellite studies (Ohsawa et al., 2007) also revealed very low levels of between-population differentiation and withinpopulation inbreeding for all populations examined.

\section{Nucleotide variation}

Standard statistics of nucleotide variation for the three loci, MetE, Cons109 and Cons14, in each population and the pool (combined sequence data) are presented in Table 2. A total of 510 sequences was sampled for the three loci in nine populations, thus an average of $\sim 19$ sequences per locus per population were used to estimate nucleotide polymorphism $\left(\theta_{\mathrm{W}}\right)$ as well as nucleotide diversity at total sites $\left(\pi_{\text {tot }}\right)$ and at silent sites $\left(\pi_{\mathrm{sil}}\right)$. The levels of nucleotide diversity are heterogeneous among loci, with MetE being the least diverse locus $\left(\pi_{\text {tot }}=0.00667, \pi_{\text {sil }}=0.00711\right.$ in the pool $)$ and Cons109 being the highest $\left(\pi_{\mathrm{tot}}=0.00721, \pi_{\mathrm{sil}}=0.00768\right.$ 
Table 2 Summary of nucleotide variation for MetE, Cons109 and Cons14

\begin{tabular}{|c|c|c|c|c|c|c|c|c|c|c|}
\hline Population & Locus & $\mathrm{n}^{\mathrm{a}}$ & $S^{b}$ & $h^{c}$ & $\pi_{t o t}^{\mathrm{d}}$ & $\pi_{s i l}^{\mathrm{d}}$ & $\theta_{W}^{\mathrm{d}}$ & Tajima's $D^{\mathrm{e}}$ & $F u$ and $L i^{\prime} s D^{\mathrm{e}}$ & $F u$ and $L i^{\prime} s \mathrm{~F}^{\mathrm{e}}$ \\
\hline \multirow[t]{3}{*}{ N1 } & MetE & 14 & 29 & 13 & 587 & 621 & 662 & -0.4843 & -0.1927 & -0.3123 \\
\hline & Cons109 & 26 & 28 & 16 & 699 & 710 & 722 & -0.3180 & -0.5670 & -0.7680 \\
\hline & Cons14 & 20 & 29 & 20 & 678 & 722 & 743 & -0.1120 & -0.5980 & -0.8650 \\
\hline \multirow[t]{3}{*}{ N2 } & MetE & 14 & 30 & 12 & 637 & 699 & 685 & -0.2972 & -0.4497 & -0.4684 \\
\hline & Cons109 & 24 & 26 & 19 & 673 & 987 & 687 & -0.6570 & -0.8310 & -0.4790 \\
\hline & Cons14 & 20 & 29 & 19 & 735 & 766 & 743 & -0.2110 & -0.8790 & -0.1210 \\
\hline \multirow[t]{3}{*}{ N3 } & MetE & 14 & 28 & 9 & 679 & 632 & 639 & 0.2656 & 0.5876 & 0.5736 \\
\hline & Cons109 & 20 & 28 & 14 & 721 & 746 & 777 & -0.6510 & -0.8590 & -0.4200 \\
\hline & Cons14 & 20 & 28 & 17 & 699 & 710 & 717 & -1.1300 & -1.2760 & -1.3400 \\
\hline \multirow[t]{3}{*}{ N4 } & MetE & 12 & 26 & 8 & 792 & 799 & 625 & 1.1926 & 0.7400 & 0.9778 \\
\hline & Cons109 & 20 & 27 & 19 & 745 & 763 & 750 & -0.0470 & -0.5690 & -0.7310 \\
\hline & Cons14 & 20 & 25 & 16 & 732 & 785 & 641 & 1.1230 & 1.1100 & 0.9710 \\
\hline \multirow[t]{3}{*}{ N5 } & MetE & 12 & 25 & 7 & 588 & 645 & 612 & -1.2220 & -1.1140 & 1.4680 \\
\hline & Cons109 & 20 & 26 & 11 & 697 & 699 & 744 & -1.1210 & -0.9860 & -0.9920 \\
\hline & Cons14 & 20 & 24 & 14 & 677 & 756 & 633 & 1.1120 & 0.9770 & 0.9927 \\
\hline \multirow[t]{3}{*}{ S1 } & MetE & 14 & 26 & 8 & 620 & 699 & 594 & 0.1888 & -0.0353 & 0.0295 \\
\hline & Cons109 & 26 & 27 & 17 & 700 & 743 & 697 & 0.2780 & 0.7860 & 0.8710 \\
\hline & Cons14 & 24 & 25 & 15 & 614 & 675 & 609 & 0.5430 & 0.7790 & 0.9880 \\
\hline \multirow[t]{3}{*}{ S2 } & MetE & 12 & 22 & 5 & 786 & 798 & 529 & $2.1532^{*}$ & $1.3665^{*}$ & $1.7890^{*}$ \\
\hline & Cons109 & 26 & 22 & 10 & 761 & 782 & 568 & $2.0490^{*}$ & $1.7200^{*}$ & $1.9800^{*}$ \\
\hline & Cons14 & 20 & 23 & 9 & 778 & 796 & 589 & $2.0080^{*}$ & $1.7700^{*}$ & $1.6900^{*}$ \\
\hline \multirow[t]{3}{*}{ S3 } & MetE & 14 & 21 & 6 & 309 & 444 & 456 & -1.3535 & -2.1183 & -2.1900 \\
\hline & Cons109 & 24 & 25 & 12 & 598 & 652 & 660 & -0.9770 & -0.8210 & -0.9110 \\
\hline & Cons14 & 20 & 28 & 10 & 710 & 734 & 717 & -0.0120 & -0.7880 & -0.9760 \\
\hline \multirow[t]{3}{*}{ S4 } & MetE & 14 & 26 & 11 & 618 & 643 & 594 & 0.1774 & -0.3968 & -0.2759 \\
\hline & Cons109 & 20 & 24 & 13 & 691 & 678 & 666 & 0.7860 & -0.1200 & -0.2310 \\
\hline & Cons14 & 20 & 24 & 13 & 669 & 721 & 615 & 0.5980 & 0.7830 & 0.9180 \\
\hline \multirow[t]{4}{*}{ Pool $^{\mathrm{f}}$} & MetE & 120 & 66 & 56 & 667 & 711 & 889 & -0.8623 & $-3.3344^{*}$ & $-2.7100^{*}$ \\
\hline & Cons109 & 206 & 79 & 66 & 721 & 768 & 1318 & -0.9970 & -0.9210 & -0.8910 \\
\hline & Cons14 & 184 & 84 & 65 & 699 & 715 & 1319 & -0.9699 & -0.8870 & -0.5230 \\
\hline & $\mathrm{All}^{\mathrm{g}}$ & 510 & 229 & & 693 & 780 & 960 & & & \\
\hline
\end{tabular}

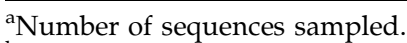

${ }^{b}$ Number of segregating sites.

'Number of haplotypes.

${ }^{\mathrm{d}}$ Parameters of nucleotide variation were multiplied by a factor of $10^{5}$.

eAn asterisk $\left(^{*}\right)$ is labeled when a statistic value is significant at a $95 \%$ confidence level $(P<0.05)$.

${ }^{\mathrm{f}}$ Combined sequence data of all 9 populations.

gequence of all 3 loci ( $3500 \mathrm{bp})$ was used for analysis.

Table 3 Summary of $\mathrm{F}_{\mathrm{ST}}$ and tests of genetic differentiation

\begin{tabular}{lccc}
\hline Locus & Pool & Southernmost populations & Northernmost populations \\
\hline MetE & $0.078^{*}$ & $0.097^{*}$ & $0.070^{*}$ \\
Cons109 & $0.034^{*}$ & $0.046^{*}$ & $0.044^{*}$ \\
Cons14 & $0.077^{*}$ & $0.072^{*}$ & $0.078^{*}$ \\
Combined 3 loci & $0.091^{*}$ & $0.087^{*}$ & $0.093^{*}$ \\
Short EST loci & $0.023^{*}(0.001-0.033)$ & $0.044^{*}(0.006-0.053)$ & $0.037^{*}(0.009-0.051)$ \\
Average of all loci & 0.053 & 0.065 & 0.057
\end{tabular}

Wright's fixation index $\left(\mathrm{F}_{\mathrm{ST}}\right)$ was estimated based on the sequence data for the 3 loci MetE, Cons109, and Cons14 and the SNP data for the 8 short loci across populations examined (9 populations for the 3 loci and 6 populations for the 8 short loci) by the method of Hudson $e t$ al. (1992) and with FSTAT, respectively. Probabilities of the statistical test of genetic differentiation $(P)$ were estimated based on Hudson's (2000) Snn statistic, evaluated by 10000 permutations for the 3 loci; significance of $F_{\text {ST }}$ for the 8 short loci was evaluated with FSTAT (1000 randomizations). The numbers in parentheses indicate $99 \%$ confidence intervals of $\mathrm{F}_{\mathrm{ST}}$; an asterisk $\left(^{*}\right)$ indicates statistical significance at a $95 \%$ confidence level $(P<0.05)$.

in the pool). When the sequence data of all three loci are combined, $\pi_{\text {tot }}=0.00693, \pi_{\text {sil }}=0.00780$ and $\theta_{\mathrm{W}}=0.00961$ in the pool. As shown in Table $4, \pi$ was also estimated from the eight short loci based on their SNP data. Because of the short sequence lengths, we combined the data of all the eight loci to estimate $\pi$ at total sites (2529 bp). A total of 20 sequences per locus per population were used for the estimation. In the pool of six populations, $\pi_{\text {tot }}$ was estimated to be 0.00598
$( \pm 0.00100$; Table 4). This value is quite similar to that estimated from the combined sequence data of the three long loci mentioned above.

Being more recently colonized, the northern Japan populations are expected to have a lower level of genetic diversity than the southern populations. However, our calculations showed that nucleotide diversity in the three northernmost populations (N1-N3) was not lower than that in the three southernmost populations (S2-S4) for all 
Table 4 Summary of nucleotide variation in the eight short EST loci

\begin{tabular}{|c|c|c|c|c|c|c|c|}
\hline Population & N1 & N2 & N3 & S1 & S2 & S3 & Pool \\
\hline$\pi^{\mathrm{a}}$ (s.d.) & 618.72 (1.73) & $655.14(0.49)$ & $618.93(2.38)$ & $570.44(3.28)$ & $564.00(3.82)$ & $578.10(3.84)$ & $598.15(1.00)$ \\
\hline $\mathrm{HKA}^{\mathrm{b}}$ & 0.991 & 0.932 & 0.897 & 0.954 & 0.939 & 0.984 & 0.933 \\
\hline
\end{tabular}

${ }^{\mathrm{a}} \pi$ and its standard deviation (s.d.) were multiplied by a factor of $10^{5}$.

${ }^{b}$ Probability of the multilocus HKA test (Hudson et al., 1987) for each population and the pool.

Table 5 Summary of levels of recombination

\begin{tabular}{lcccccccrrr}
\hline & $N 1$ & $N 2$ & $N 3$ & $N 4$ & $N 5$ & $S 1$ & $S 2$ & $S 3$ & S4 & Pool \\
\hline$R_{\mathrm{M}}$ a & 9 & 9 & 9 & 12 & 2 & 2 & 3 & 1 & 4 \\
No. of informative sites & 54 & 51 & 66 & 63 & 57 & 51 & 63 & 15 & 45 \\
$R_{\mathrm{M}}$ /no. of informative sites & 0.20 & 0.18 & 0.14 & 0.19 & 0.04 & 0.04 & 0.05 & 0.07 & 0.09 & 0.32 \\
\hline
\end{tabular}

${ }^{\mathrm{a}} R_{\mathrm{M}}$, the minimum number of recombination events (Hudson and Kaplan, 1985).

three loci, MetE, Cons109 and Cons14 as well as the short loci (Table 4); it is also clearly seen in Tables 2 and 3 that all genetic diversity parameters, that is, $\pi_{\mathrm{tot}}, \pi_{\mathrm{sil}}$ and $\theta_{\mathrm{W}}$ are generally not lower in the northern populations than in the southern populations.

\section{Recombination and linkage disequilibrium}

In this article, we calculated the minimum number of recombination events, $R_{\mathrm{M}}$, per informative site estimated from the combined data of all the three loci, MetE, Cons109 and Cons14 (3500 bp), to compare the level of recombination among populations. As shown in Table 5, $R_{\mathrm{M}}$ per informative site for the three loci was 0.32 in the pool, and ranged from 0.04 to 0.20 among populations. The levels of recombination appeared higher in the four northernmost populations (N1-N4) than in the remaining populations, despite that the statistical test supported a significant or marginally significant difference with N5, S1 and S2 populations only $(P<0.10$, two-tailed Fisher's exact test).

In theory, when recombination drives allelic diversity, LD between polymorphic sites is expected to decline with increasing distance along the chromosome. To understand this relationship, we compared the number of significant comparisons among sites $>400 \mathrm{bp}$ apart and $<400 \mathrm{bp}$ apart for each locus in the pool. The results of all three loci showed that both the average values of $r^{2}$ and $\left|D^{\prime}\right|$ were significantly larger among sites $<400 \mathrm{bp}$ apart, and the percentage of significant pairwise comparison was also significantly higher $(P<0.05)$ in this group (Figure 2; Supplementary Tables S2, S3 and S4), suggesting that on average, LD significantly declined among sites $>400 \mathrm{bp}$ apart in species-wide samples. However, LD did not decline significantly among sites $>400 \mathrm{bp}$ apart within populations, except for the three northernmost populations (N1-N3; Figure 2; Supplementary Tables S2, S3 and S4). The patterns of LD decay were similar when the low-frequency alleles $(<10 \%)$ were included or excluded from the analysis.

\section{Neutrality tests}

To examine the fit of nucleotide polymorphism data to the neutral equilibrium model, we performed the tests of Tajima's D (Tajima, 1989a) and Fu and Li's D and F
( $\mathrm{Fu}$ and $\mathrm{Li}, 1993$ ) for each locus in each population separately and in the pool of nine populations. No significant Tajima's $D$ and $\mathrm{Fu}$ and $\mathrm{Li}^{\prime} \mathrm{s} D$ and $\mathrm{F}$ were observed at any of the three loci except for MetE in the pool ( $\mathrm{Fu}$ and Li's $D$ and $\mathrm{F}$ were significantly negative) and all the three loci in population S2 (Tajima's D and Fu and Li's $D$ and F were all significantly positive; Table 2). It is interesting to note that Tajima's $D$ is negative at all the three loci in the pool but positive in three out of four populations in southern Japan (S1, S2 and S4). The multilocus HKA test was used to assess departures from the neutral model for the eight short EST loci. We observed no evidence for any significant deviations in any population and in the pool $(P>0.05$; Table 4$)$.

\section{Discussion}

\section{Nucleotide variation}

The occurrence frequency of SNP in our samples of $Q$. crispula is 1 every $\sim 25 \mathrm{bp}$, which is comparable to that of other plant species previously reported. For example, $1 \mathrm{SNP}$ every $\sim 50 \mathrm{bp}$ was detected when the average of 1095 randomly selected gene fragments in maize was considered (Yamasaki et al., 2005); a frequency of 1 every $23 \mathrm{bp}$ was scored when the average of 10 unlinked loci in rice was taken (Zhu et al., 2007); a frequency of 1 every $60 \mathrm{bp}$ was obtained when the average of 5 loci in European aspen was considered (Ingvarsson, 2005). This is one of the first studies describing the level of nucleotide polymorphism in oak species. The LD mapping approach requires abundant SNPs in the target species. In this study, SNPs were detected in all gene fragments, even in those with only a couple of hundred base pairs in length. Thus, screening for adaptive variation in oaks can be efficient with short sequences by the genome-wide scan approach as performed in maize (Yamasaki et al., 2005).

The present study is an additional contribution to increase knowledge about nucleotide diversity in forest tree species. We use both $\pi_{\text {tol }}$ and $\pi_{\text {sil }}$ to compare the levels of nucleotide diversity among species for the following discussion because $\pi_{\text {sil }}$ has not yet been reported for several tree species in previous studies (Supplementary Table S5). Nucleotide diversity is 

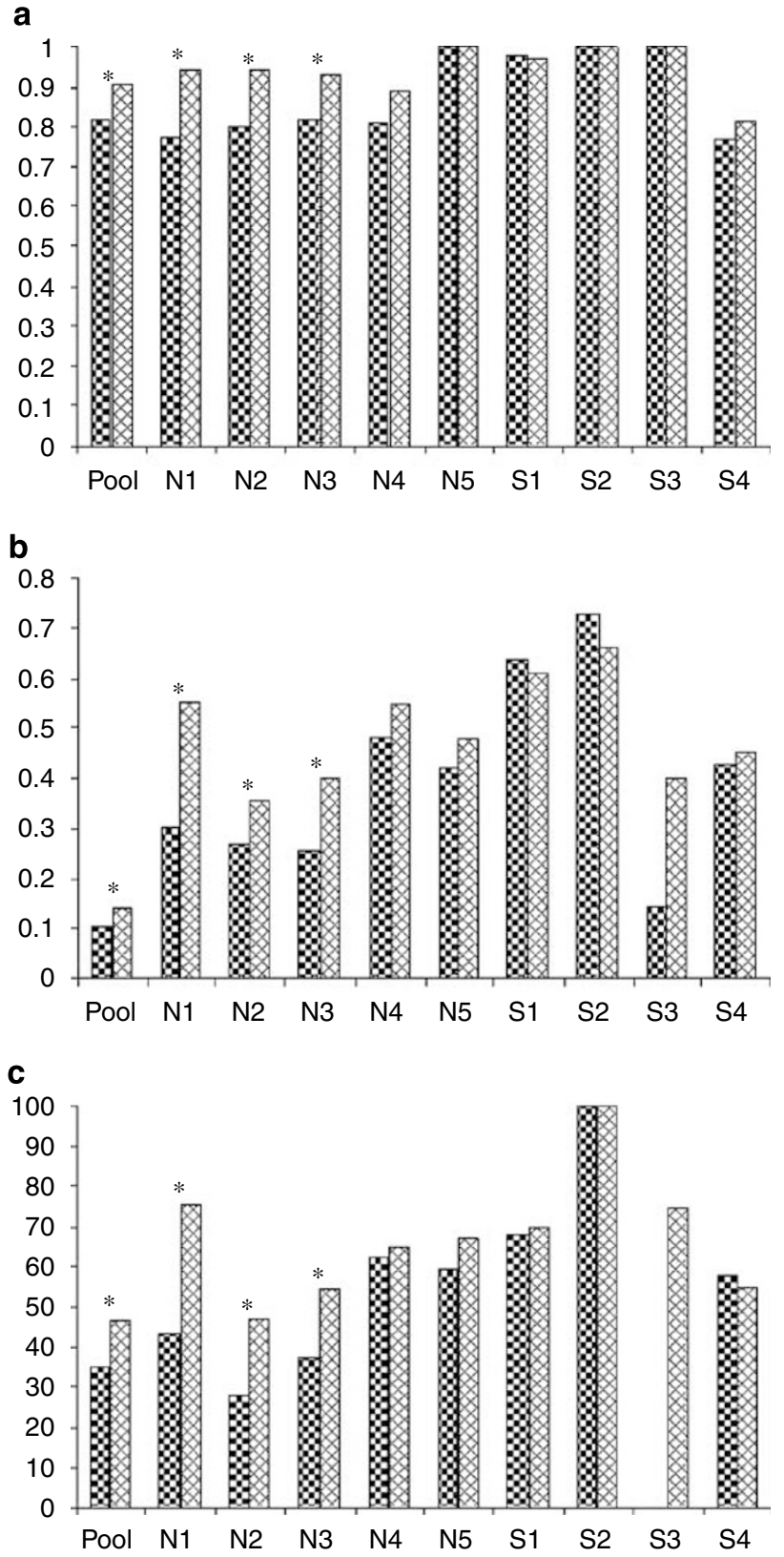

Figure 2 LD analysis of the species-wide sample (Pool) and different local populations (N1-S4) at the MetE locus. Average values estimated among sites $>400 \mathrm{bp}$ apart and $<400 \mathrm{bp}$ apart are indicated by the first and second columns, respectively, for the pool and for each population separately; (a) comparisons of $\left|D^{\prime}\right|$, (b) comparisons of $r^{2}$ and (c) comparisons of percentage of significant pairwise tests; when the difference between the two groups is statistically significant $(P<0.05)$, an asterisk $(*)$ is labeled.

generally low in conifers such as Pinus, Cryptomeria and Chamaecyparis but relatively high in broadleaved trees such as Populus and Quercus. The nucleotide diversity of $Q$. crispula is generally higher than that of conifers, as high as that of a European oak, but lower than that of the European aspen (Supplementary Table S5).

Besides the mating system and life history traits, demographic history has a major impact on the level of within-population genetic diversity (Hamrick and Godt, 1996; Charlesworth, 2003). The results of this study imply that the northernmost populations do not harbor lower nucleotide diversity than the southernmost populations. This is interesting given the recent colonization history of the northern Japan populations. Theoretical models showed that the recently colonized populations are generally expected to show a decline in genetic diversity due to genetic bottlenecks and founder events (Slatekin, 1977; Pannell and Charlesworth, 1999; Pannell and Dorken, 2006). Possible alternative hypotheses will be discussed below together with population recombination and LD decay.

\section{Mating system and population structures}

Given the differences in the current patterns of population distribution and the density of the forests in northern and southern Japan, the level of inbreeding may be expected to be higher in the southern populations at some degree. Nevertheless, inbreeding indices were not significantly different from zero in all the populations sampled.

The average $\mathrm{F}_{\mathrm{ST}}$ was estimated to be 0.053 in this study; the equivalent measurement of $\mathrm{F}_{\mathrm{ST}}$ was 0.013 as estimated from microsatellite data in a previous study (Ohsawa et al., 2007). Even though these two $\mathrm{F}_{\mathrm{ST}}$ estimates were substantially different, which was probably due to sampling strategies (latitudinal gradient along Japan vs altitudinal gradient in a narrow latitudinal range) and the genetic nature (biallelic vs multiple allelic polymorphism and evolutionary mechanisms) of the markers, both studies consistently revealed a modest level of genetic differentiation among populations. $Q$. crispula is an obligate outcrossing and wind-pollinated long-lived forest tree and has seeds that may be dispersed over a long distance by birds and rodents (Watanabe, 1994). Hence, the low levels of population differentiation observed among populations of this species are consistent with a general observation for outbreeding and wind-pollinated species (Hamrick and Godt, 1990). Nonetheless, low F $F_{S T}$ was unexpectedly observed among the recently colonized populations (northernmost populations). Several theoretical studies showed that colonization frequently leads to an excess of differentiation among populations compared with the equilibrium situation (Austerlitz et al., 1997; Le Corre and Kremer, 1998); however, with time, genetic differentiation is expected to decrease because of migration between extant populations (Pannell and Dorken, 2006).

Tajima's $D$-values of all the three loci were negative in the pool but positive or even significantly positive in several southern Japan populations. This suggests an excess of low-frequency polymorphisms in the specieswide samples and an excess of intermediate-frequency polymorphisms in the populations from southern Japan. The excess of low-frequency or intermediate-frequency polymorphisms is consistent across three loci, thus natural selection seems not to be the evolutionary force causing these skews in the frequency spectrum. The excess of intermediate-frequency polymorphisms in the populations from the south appears to be a genetic consequence of a recent reduction in population size (Tajima, 1989b), because of the preferential loss of lowfrequency polymorphisms. The observation of more negative Tajima's $D$ in the pooled sample as opposed to the within-populations of $Q$. crispula in this study was 
similar to that in Populus (Ingvarsson, 2005) and wild tomatoes (Arunyawat et al., 2007). This excess of lowfrequency polymorphism can be explained by species range expansion in Japan, probably the recent northern colonization.

Furthermore, palynological studies have suggested that oak forests covered a broad area in southern Japan during LGM and have just recently reached the northern areas. In southern Japan, the frequency of oak pollen rapidly increased 12000 years ago and then began to decrease from 8500 years ago (Miyoshi, 1998; Takahara, 1998). However, in a population from southern Japan (S3), we did not observe the excess of intermediatefrequency polymorphisms; this may suggest that either no such significant reduction in population size occurred in this population or singleton mutations have been reaccumulated in this population owing to gene flow from other populations within a time period of approximately 8500 years.

\section{Recombination and linkage disequilibrium}

We showed that the level of recombination was relatively high in $Q$.crispula as $R_{M}$ per informative site was 0.32 for the species-wide samples. The values of $R_{\mathrm{M}}$ per informative site at seven loci in Arabidopsis thaliana were lower and ranged from 0.08 to 0.15 (Kuittinen and Aguage, 2000); however, these values were still much higher than those in Cryptomeria japonica, which ranged from 0.0000 to 0.0526 (Kado et al., 2003); $R_{\mathrm{M}}$ per informative site in the Pal1 locus of Pinus sylvestris was 0.25 (Dvornyk et al., 2002). Note that A. thaliana is mostly selfing and thus seems to have very low effective recombination rates (Gaut and Long, 2003). The recombination rate in C. japonica was about one-thirtieth of that in A. thaliana on average (Kado et al., 2003). P. sylvestris is among the tree species with the widest-known geographic distributions in the world, whereas Q. crispula is restricted to some East Asian areas; however, the levels of both nucleotide diversity and population recombination were generally higher in this oak than the conifer. This is attributable to the higher mutation and recombination rates in oaks than in conifers. Alternatively, past and/or ongoing hybridization among closely related oak species, such as between $Q$. crispula and $Q$. dentata (Ishida et al., 2003) and between $Q$. crispula and Q. serrata (Kanazashi et al., 1997) in Japan may be one factor maintaining the high levels of nucleotide variation and recombination in oaks.

This study together with a previous study (Ingvarsson, 2005) showed that broadleaved trees such as oaks and aspens may show generally higher levels of species-wide nucleotide diversity and recombination than conifers. Theoretical and empirical studies have shown that the mating system primarily affects diversity at the population level rather than at the species level (Savolainen et al., 2000; Ingvarsson, 2002; Charlesworth, 2003; Wright et al., 2003). Oaks, aspens and conifers are all mostly outcrossing long-lived trees. Thus, differences in life history traits might be an important factor responsible for such different levels of nucleotide diversity and recombination between broadleaved and coniferous species.

Perhaps, one of our most interesting findings is that the three northernmost populations harbor a relatively large amount of nucleotide diversity, higher levels of recombination and faster LD decay than the southern populations in Japan. This result is contradictory to theoretical expectations given the recent colonization history of the northern Japan populations. Several possibilities should be considered to explain this paradox. The first is that effective population size is larger in the recently colonized populations than in the southern populations due to a recent population expansion that occurred in the former and/or a recent population bottleneck in the latter. Statistical tests and palynological data appear to favor the hypothesis of a recent population bottleneck in southern Japan populations.

A second possibility could be natural selection. Purifying selection reduces genetic diversity and the southern populations are more intensively selected than the northern populations; the LD decay was shown to be significantly slower in the former than in the latter, thereby causing hitchhiking and background selection more effective (Kaplan et al., 1989; Begun and Aquadro, 1992; Charlesworth et al., 1993). However, the possibility of selection is not supported by statistical tests in this study. It should be noted that, because of the generally low statistical power of most tests (Wright and Gaut, 2005), the effects of selection cannot be excluded entirely for all loci.

The relatively high levels of nucleotide polymorphism and recombination in the recently colonized populations in northern Japan may also be explained by several possibilities. Firstly, gene flow subsequent to colonization might be sufficiently strong to increase genetic diversity in the populations when they reach demographic equilibrium (Pannell and Dorken, 2006). Secondly, the long lifespan and juvenile phase of trees together with long-distance seed dispersal were shown to strongly attenuate founder effects during colonization (Austerlitz et al., 1997; Austerlitz and Garnier-Gere, 2003). Lastly, multiple colonization events causing an admixture of different lineages may overcome bottlenecks in recently colonized populations, particularly when propagules have long residence time or retain high genetic diversity as do the acorns of oaks (Lavergne and Molofsky, 2007).

Although further studies are needed to test each hypothesis mentioned above, the current landscape of genetic diversity, recombination and LD in the natural populations of $Q$. crispula characterized in this study may provide primarily information for conservation genetics and LD mapping studies for this species and facilitate molecular evolution studies in oaks.

\section{Acknowledgements}

We thank Professor John Pannell (Oxford University), Dr NM Tue (CMES, Ehime University), the subject editor and three anonymous referees for their helpful discussion and critical reading of our article. This research was supported by a Grant-in-Aid (No. 16380104) from the Japan Society for the Promotion of Science.

\section{References}

Aguade M (2001). Nucleotide sequence variation at two genes of the phenylpropanoid pathway, the $F A H 1$ and $F 3 H$ genes, in Arabidopsis thaliana. Mol Biol Evol 18: 1-9. 
Arunyawat U, Stephant W, Städler T (2007). Using multilocus sequence data to assess population structure, natural selection, and linkage disequilibrium in wild tomatoes. Mol Biol Evol 24: 2310-2322.

Austerlitz F, Garnier-Gere PH (2003). Modelling the impact of colonization on genetic diversity and differentiation of forest trees: interaction of life cycle, pollen flow and seed longdistance dispersal. Heredity 90: 282-290.

Austerlitz F, Jung-Muller B, Godelle B, Gouyon PH (1997). Evolution of coalescence times, genetic diversity and structure during colonization. Theor Popul Biol 51: 148-164.

Begun D, Aquadro CF (1992). Levels of naturally occurring DNA polymorphism correlate with recombination rates in $D$. melanogaster. Nature 356: 519-529.

Casasoli M, Derory J, Morera-Dutrey C, Brendel O, Porth I, Guehl JM et al. (2006). Comparison of quantitative trait loci for adaptive traits between oak and chestnut based on an expressed sequence tag consensus map. Genetics 172: 533-546.

Charlesworth B, Morgan TH, Charlesworth D (1993). The effect of deleterious mutations on neutral molecular variation. Genetics 134: 1289-1303.

Charlesworth D (2003). Effects of inbreeding on the genetic diversity of populations. Philos Trans R Soc Lond Biol B Biol Sci 358: 1051-1070.

Chiang Y, Ge CXJ, Chou CH, Wu WL, Chiang TY (2002). Nucleotide sequence diversity at the methionine synthase locus in endangered Dunnia sinensis (Rubiaceae): an evaluation of the positive selection hypothesis. Mol Biol Evol 19: 1367-1375.

Dvornyk V, Sirvio A, Mikkonen M, Savolainen O (2002). Low nucleotide diversity at the pal1 locus in the widely distributed Pinus sylvestris. Mol Biol Evol 19: 179-188.

Filatov DA, Charlesworth D (1999). DNA polymorphism, haplotype structure and balancing selection in the Leavenworthia PgiC locus. Genetics 147: 915-925.

$\mathrm{Fu} \mathrm{YX,} \mathrm{Li} \mathrm{WH} \mathrm{(1993).} \mathrm{Statistical} \mathrm{tests} \mathrm{of} \mathrm{neutrality} \mathrm{of} \mathrm{mutatons.}$ Genetics 133: 693-709.

Garcia-Gil MR, Mikkonen M, Savolainen O (2003). Nucleotide diversity at two phytochrome loci along a latitudinal cline in Pinus sylvestris. Mol Ecol 12: 1195-1206.

Gaut BS, Long AD (2003). The lowdown on linkage disequilibrium. Plant Cell 15: 1502-1506.

Goudet J (2001). FSTAT (version 2.9.3): a program to estimate and tests gene diversities and fixation indices. http:// www2.unil.ch/popgen/softwares/fstat.htm.

Hamrick JL, Godt MJW (1990). Allozyme diversity in plant species. In: Brown AHD, Clegg MT, Kahler AL and Weir BS (eds). Plant Population Genetics, Breeding and Genetic Resources. Sinauer Associates: Sunderland, MA.pp 43-63.

Hamrick JL, Godt MJW (1996). Effects of life history traits on genetic diversity in plant species. Philos Trans $R$ Soc Lond $B$ Biol Sci 351: 1291-1298.

Hibino K, Takeuchi S (1998). Vegetation history of the Tohoku district. In: Yasuda Y and Miyoshi N (eds). Illustration: Vegetation History of the Japanese Archipelago. Asakura-shoten: Tokyo, Japan. (in Japanese) 62-72.

Horikawa Y (1972). Atlas of the Japanese flora, an Introduction to Plant Sociology of East Asia. Gakken Co. Ltd: Tokyo, Japan.

Hudson RR (2000). A new tests statistic for detecting genetics differentiation. Genetics 155: 2011-2014.

Hudson RR, Boos DD, Kaplan NL (1992). A statistical test for detecting geographic subdivision. Mol Biol Evol 9: 138-151.

Hudson RR, Kaplan NL (1985). Statistical properties of the number of recombination events in the history of a sample of DNA sequences. Genetics 111: 147-164.

Hudson RR, Kreitman M, Aguade M (1987). A tests of neutral molecular evolution based on nucleotide data. Genetics 116: 153-159.

Ingvarsson P (2005). Nucleotide polymorphism and linkage disequilibrium within and among natural populations of
European aspen (Populus tremula L. Salicaceae). Genetics 169: 945-953.

Ingvarsson PK (2002). A metapopulation perspective of genetic diversity and differentiation in partially self-fertilizing plants. Evolution 56: 2368-2373.

Innan H, Tajima F, Terauchi R, Miyashita T (1996). Intragenic recombination in the Adh locus of the wild plant Arabidopsis thaliana. Genetics 143: 1761-1770.

Ishida TA, Hattori K, Sato H, Kimura MA (2003). Differentiation and hybridization between Quercus crispula and $Q$. dentate (Fagaceae): insights from morphological traits, amplified fragment length polymorphism markers, and leafminer composition. Am J Bot 90: 769-776.

Kado T, Matsumoto A, Ujino-Ihara T, Tsumura Y (2008). Amounts and patterns of nucleotide variation within and between two Japanese conifers, sugi (Cryptomeria japonica) and hinoki (Chamaecyparis obtuse) (Cupressaceae sensu lato). Tree Genet Genomes 4: 133-141.

Kado T, Yoshimaru H, Tsumura Y, Tachida H (2003). DNA variation in a conifer, Cryptomeria japonica (Copressaceae sensu lato). Genetics 164: 1547-1599.

Kanazashi A, Kanazashi T, Kawahara T (1997). Evidence of reproductive barriers between closely related species of Quercus. In: Steiner KC (ed). Diversity and Adaptation in Oak Species. Proc 2nd Meeting of Working Party 2.08.05. Genetics Quercus IUFRO.

Kanno M, Yokoyama J, Suyama S, Ohyama M, Itoh T, Suzuki M (2004). Geographical distribution of two haplotypes of chloroplast DNA in four oak species (Quercus) in Japan. J Plant Res 117: 311-317.

Kaplan NL, Hudson RR, Langley CH (1989). The 'hitchhiking effect' revisited. Genetics 123: 887-899.

Kubitzki K (1993). Fagaceae. In: Kubitzki K, Rohwer JG, Bittrich V (eds). The Families and Genera of Vascular Plants, Vol. 2. Springer: Berlin. pp 301-309.

Kuittinen H, Aguage M (2000). Nucleotide variation at the chalcone isomerase locus in Arabidopsis thaliana. Genetics 155: 863-872.

Lavergne S, Molofsky J (2007). Increased genetic variation and evolutionary potential drive the success of an invasive grass. Proc Natl Acad Sci USA 10: 3883-3888.

Le Corre V, Kremer A (1998). Cumulative effects of founding events during colonization on genetic diversity and differentiation in an island and stepping stone model. J Ecol Biol 11: $495-512$.

Miyoshi N (1998). Vegetation History of the Chugoku and Shikoku districts. In: Yasuda $\mathrm{Y}$ and Miyoshi $\mathrm{N}$ (eds). Illustration: Vegetation History of the Japanese Archipelago. Asakura-shoten: Tokyo, Japan. (in Japanese) 138-149.

Morita Y, Choi K, Hibino K (1998). Vegetation history of the Chubu and Tokai districts. In: Yasuda Y and Miyoshi N (eds). Illustration: Vegetation History of the Japanese Archipelago. Asakura-shoten: Tokyo, Japan. (in Japanese) 92-102.

Neale DB, Savolainen O (2004). Association genetics of complex traits in conifers. Trends Plant Sci 9: 325-330.

Nixon KC (1993). Infrageneric classification of Quercus (Fagaceae) and typification of sectional names. Ann Sci Forest $\mathbf{5 0}$ (Suppl. 1): 25s-34s.

Ohsawa T, Tsuda Y, Saito Y, Sawada H, Ide Y (2007). Altitudinal genetic diversity and differentiation of Quercus crispula in the Chichibu mountains, central Japan. Int J Plant Sci 168: 333-340.

Okaura T, Harada K (2002). Phylogeographical structure revealed by chloroplast DNA variation in Japanese Beech (Fagus crenata Blume). Heredity 88: 322-329.

Okaura T, Quang ND, Ubukata M, Harada K (2007). Phylogeographic structure and late quaternary population history of the Japanese oak Quercus mongolica var. crispula and related species revealed by chloroplast DNA variation. Genes Genet Syst 82: 465-477. 
Pannell JR, Charlesworth B (1999). Neutral genetic diversity in a metapopulation with recurrent local extinction and recolonization. Evolution 53: 664-676.

Pannell JR, Dorken ME (2006). Colonisation as a common denominator in plant metapopulations and range expansions: effects on genetic diversity and sexual systems. Landscape Ecol 21: 837-848.

Pot D, McMillan L, Echt C, Le Provost G, Garnier-Gere P, Cato S et al. (2005). Nucleotide variation in genes involved in wood formation in two pine species. New Phytol 167: 101-112.

Remington DL, Thornsberry JM, Matsoura Y, Wilson LM, Whitt SR, Doebley J et al. (2001). Structure of linkage disequilibrium and phenotypic associations in the maize genome. Proc Natl Acad Sci USA 98: 11479-11484.

Rieseberg LH, Wendel JF (1993). Introgression and its consequences in plants. In: Harrison RG (ed). Hybrid Zones and the Evolutionary Process. Oxford University Press: Oxford. pp 70-109.

Rozas J, Sánchez-DelBarrio JC, Messeguer X, Rozas R (2003). DnaSP, DNA polymorphism analyses by the coalescent and other methods. Bioinformatics 19: 2496-2497.

Savolainen O, Langley CH, Lazzaro BP, Fr H (2000). Contrasting patterns of nucleotide polymorphism at the alcohol dehydrogenase locus in the outcrossing Arabidopsis lyrata and the selfing Arabidopsis thaliana. Mol Biol Evol 17: 645-655.

Slatekin M (1977). Gene flow and genetic drift in a species subject to frequent local extinctions. Theor Popul Biol 12: 253-262.

Tajima F (1989a). Statistical methods for testing the neutral mutation hypothesis by DNA polymorphism. Genetics 123: 585-595.
Tajima F (1989b). The effect of change in population size on DNA polymorphism. Genetics 123: 597-601.

Takahara H (1998). Vegetation history of the Kinki district. In: Yasuda Y and Miyoshi N (eds). Illustration: Vegetation History of the Japanese Archipelago. Asakura-shoten: Tokyo, Japan. (in Japanese) 114-134.

Van VL (1976). Ecological species, multispecies, and oaks. Taxon 2-3: 769-776.

Watanabe S (1994). Specia of Trees. Tokyo University Press: Tokyo. (in Japanese) 450 pp.

Wright SI, Gaut BS (2005). Molecular population genetics and the search for adaptive evolution in plants. Mol Biol Evol 22: 506-519.

Wright SI, Lauga B, Charlesworth D (2003). Subdivision and haplotype structure in natural populations of Arabidopsis lyrata. Mol Ecol 12: 1247-1263.

Yamasaki M, Tenaillon MI, Bi IV, Schroeder SG, Sanchez-Villeda H, Doebley JF et al. (2005). A large-scale screen for artificial selection in maize identifies candidate agronomic loci for domestication and crop improvement. Plant Cell 17: 2859-2872.

Zhang L-B, Ge S (2006). Multiple analysis of nucleotide variation and speciation in Oryza officinalis and its close relatives. Mol Biol Evol 24: 769-783.

Zhu Q, Zheng X, Luo J, Gaut BS, Ge S (2007). Multilocus analysis of nucleotide variation of Oryza sativa and its wild relatives: Severe bottleneck during domestication of rice. Mol Biol Evol 24: 875-888.

Supplementary Information accompanies the paper on Heredity website (http://www.nature.com/hdy) 